\title{
THE DISTRIBUTION OF IRREDUCIBLE POLYNOMIALS IN SEVERAL INDETERMINATES OVER A FINITE FIELD
}

\author{
by STEPHEN D. COHEN
}

(Received 22nd February 1967)

\section{Introduction}

We consider non-zero polynomials $f\left(x_{1}, \ldots, x_{k}\right)$ in $k$ variables $x_{1}, \ldots, x_{k}$ with coefficients in the finite field $G F[q]$ ( $q=p^{n}$ for some prime $p$ and positive integer $n$ ). We assume that the polynomials have been normalised by selecting one polynomial from each equivalence class with respect to multiplication by non-zero elements of $G F[q]$. By the degree of a polynomial $f\left(x_{1}, \ldots, x_{k}\right)$ will be understood the ordered set $\left(m_{1}, \ldots, m_{k}\right)$, where $m_{i}$ is the degree of $f\left(x_{1}, \ldots, x_{k}\right)$ in $x_{i}(i=1,2, \ldots, k)$. The degree $\left(m_{1}, \ldots, m_{k}\right)$ of a polynomial will be called totally positive if $m_{i}>0, i=1,2, \ldots, k$.

We investigate here the number of normalised irreducible polynomials distributed among all the normalised polynomials $f\left(x_{1}, \ldots, x_{k}\right)$ of degree $\left(m_{1}, \ldots, m_{k}\right)$. Let $N\left(m_{1}, \ldots, m_{k}\right)$ denote the number of normalised polynomials of degree $\left(m_{1}, \ldots, m_{k}\right)$ in $k$ variables $x_{1}, \ldots, x_{k}$ and $M\left(m_{1}, \ldots, m_{k}\right)$ the corresponding number of irreducibles. When $k=1, M(m)$ can be evaluated explicitly and is given by

$$
M(m)=\frac{1}{m} \sum_{r s=m} \mu(r) q^{s},
$$

where $\mu(r)$ is the Möbius function. It follows from (1.1) that

$$
M(m) \sim \frac{1}{m} q^{m}=\frac{1}{m} N(m), \quad(m \rightarrow \infty),
$$

for fixed $q$. More generally, Carlitz (1) has proved that, if $k \geqq 1, N_{k}(m)$ is the number of normalised polynomials in $x_{1}, \ldots, x_{k}$ which split into $m$ linear factors in some extension of $G F[q]$ and in which $x_{k}^{m}$ actually appears, and $M_{k}(m)$ is the corresponding number of irreducible polynomials, then $N_{k}(m)$ and $M_{k}(m)$ are given by

and

$$
N_{k}(m)=q^{k m}
$$

$$
M_{k}(m)=\frac{1}{m} \sum_{r s=m} \mu(r) q^{k m}
$$

Hence

$$
M_{k}(m) \sim \frac{1}{m} N_{k}(m), \quad(m \rightarrow \infty)
$$

E.M.S.-A 
holds for all $k \geqq 1$ and fixed $q$. When $k \geqq 2$ and we consider all polynomials of degree $\left(m_{1}, \ldots, m_{k}\right)$, the situation is different and much more difficult. In this case, no explicit formula corresponding to (1.1) or (1.3) is available. Carlitz (2) has shown that, in contrast to (1.2) and (1.4), when $k \geqq 2$, in a certain sense, " almost all " polynomials are irreducible. Carlitz (3) has studied the case $k=2$ in greater detail. Here we obtain the corresponding results for a general $k(\geqq 2)$. We shall assume from now on that $k \geqq 2$. We shall show, for example, that for fixed $m_{1}, \ldots, m_{k-1}$, numbered so that

$$
m_{k-1}=\max _{1 \leqq i \leqq k-1} m_{i}
$$

$M\left(m_{1}, \ldots, m_{k}\right)=\left(1-q^{-n_{k}}\right) N\left(m_{1}, \ldots, m_{k}\right)+O\left(m_{k} q^{\left(m_{1}+1\right) \ldots\left(m_{k-2}+1\right) m_{k-1} m_{k}}\right)$,

where

$$
n_{k}=\left\{\prod_{t=1}^{k-1}\left(m_{t}+1\right)\right\}-1
$$

and the constant in the $O$-term is independent of $m_{k}$. We shall see later that

$$
N\left(m_{1}, \ldots, m_{k}\right)=O\left(q^{\left(m_{1}+1\right) \ldots\left(m_{k}+1\right)}\right),
$$

where the constant implied by the $O$-term depends only on $q$. We examine results like (1.5) more closely obtaining improvements in most cases. We conclude by giving some examples.

\section{Fundamental formula}

The following lemma is an extension of the corresponding result for $k=2$ in (3).

Lemma 1. We have

$m_{1} N\left(m_{1}, \ldots, m_{k}\right)=\sum_{r_{1}=0}^{m_{1}} \ldots \sum_{r_{k}=0}^{m_{k}} r_{1} L\left(r_{1}, \ldots, r_{k}\right) N\left(m_{1}-r_{1}, \ldots, m_{k}-r_{k}\right)$,

where

$$
L\left(r_{1}, \ldots, r_{k}\right)=\sum_{j 1\left(r_{1}, \ldots, r_{k}\right)} \frac{1}{j} M\left(\frac{r_{1}}{j}, \ldots, \frac{r_{k}}{j}\right)
$$

[It will be necessary to distinguish by context the degree $\left(r_{1}, \ldots, r_{k}\right)$ and the symbol $\left(r_{1}, \ldots, r_{k}\right)$ meaning the greatest common divisor of $r_{1}, \ldots, r_{k}$.]

Proof. Put

$$
F\left(m_{1}, \ldots, m_{k}\right)=\Pi f\left(x_{1}, \ldots, x_{k}\right),
$$

where the product extends over all normalised polynomials of degree $\left(m_{1}, \ldots\right.$, $m_{k}$ ); also put

$$
P\left(m_{1}, \ldots, m_{k}\right)=\Pi p\left(x_{1}, \ldots, x_{k}\right)
$$

where now the product is restricted to the normalised irreducibles of degree $\left(m_{1}, \ldots, m_{k}\right)$. If $f$ is an arbitrary polynomial of degree $\left(m_{1}, \ldots, m_{k}\right)$ and $p$ is an irreducible polynomial of degree $\left(r_{1}, \ldots, r_{k}\right)$, we may put

$$
f=p^{e} g(p \nmid g) \text {, }
$$


where $e$ is a non-negative integer. Let $\Phi\left(j_{1}, \ldots, j_{k} ; p\right)$ denote the number of normalised polynomials of degree $\left(j_{1}, \ldots, j_{k}\right)$ that are not divisible by $p$. Then it follows from (2.3) that

$$
F\left(m_{1}, \ldots, m_{k}\right)=\prod_{e, p} p^{e \Phi\left(m_{1}-e r_{1}, \ldots, m_{k}-e r_{k} ; p\right)},
$$

where the product is over all $e, r_{1}, \ldots, r_{k}$ and all irreducibles $p$ of degree $\left(r_{1}\right.$, $\ldots, r_{k}$ ) such that $e r_{1} \leqq m_{1}, \ldots, e r_{k} \leqq m_{k}$. Moreover, it is evident from the definition of $\Phi\left(m_{1}, \ldots, m_{k} ; p\right)$ that

$$
\Phi\left(m_{1}, \ldots, m_{k} ; p\right)=N\left(m_{1}, \ldots, m_{k}\right)-N\left(m_{1}-r_{1}, \ldots, m_{k}-r_{k}\right),
$$

provided $m_{1} \geqq r_{1}, \ldots, m_{k} \geqq r_{k}$; otherwise

Thus (2.5) becomes

$$
\Phi\left(m_{1}, \ldots, m_{k} ; p\right)=N\left(m_{1}, \ldots, m_{k}\right) \text {. }
$$

where

$$
F\left(m_{1}, \ldots, m_{k}\right)=\prod_{r_{1}=0}^{m_{1}} \ldots \prod_{r_{k}=0}^{m_{k}} \prod p^{w},
$$

$$
\begin{aligned}
w & =\sum_{e} e \Phi\left(m_{1}-e r_{1}, \ldots, m_{k}-e r_{k} ; p\right) \\
& =\left\{N\left(m_{1}-r_{1}, \ldots, m_{k}-r_{k}\right)-N\left(m_{1}-2 r_{1}, \ldots, m_{k}-2 r_{k}\right)\right\} \\
& +2\left\{N\left(m_{1}-2 r_{1}, \ldots, m_{k}-2 r_{k}\right)-N\left(m_{1}-3 r_{1}, \ldots, m_{k}-3 r_{k}\right)\right\} \\
& +\ldots+h N\left(m_{1}-h r_{1}, \ldots, m_{k}-h r_{k}\right),
\end{aligned}
$$

where $h$ is the largest integer such that $h r_{1} \leqq m_{1}, \ldots, h r_{k} \leqq m_{k}$. Thus

so that (2.6) becomes

$$
w=\sum_{j=1}^{h} N\left(m_{1}-j r_{1}, \ldots, m_{k}-j r_{k}\right)
$$

$$
F\left(m_{1}, \ldots, m_{k}\right)=\prod_{r_{1}=0}^{m_{1}} \ldots \prod_{r_{k}=0}^{m_{k}}\left(P\left(r_{1}, \ldots, r_{k}\right)\right)^{w},
$$

with $w$ defined by (2.7) and $P\left(r_{1}, \ldots, r_{k}\right)$ by (2.4). Clearly the degree in $x_{1}$ of $F\left(m_{1}, \ldots, m_{k}\right)$ is $m_{1} N\left(m_{1}, \ldots, m_{k}\right)$, while the degree in $x_{1}$ of $P\left(r_{1}, \ldots, r_{k}\right)$ is $r_{1} M\left(r_{1}, \ldots, r_{k}\right)$. Hence (2.8) yields

$$
\begin{aligned}
m_{1} N\left(m_{1}, \ldots, m_{k}\right) & \\
& =\sum_{r_{1}=0}^{m_{1}} \ldots \sum_{r_{k}=0}^{m_{k}} r_{1} M\left(r_{1}, \ldots, r_{k}\right) \sum_{j=1}^{h} N\left(m_{1}-j r_{1}, \ldots, m_{k}-j r_{k}\right) \\
& =\sum_{u_{1}=0}^{m_{1}} \ldots \sum_{u_{k}=0}^{m_{k}} N\left(m_{1}-u_{1}, \ldots, m_{k}-u_{k}\right) \sum_{j \mid\left(u_{1}, \ldots, u_{k}\right)} \frac{u_{1}}{j} M\left(\frac{u_{1}}{j}, \ldots, \frac{u_{k}}{j}\right) \\
& =\sum_{u_{1}=0}^{m_{1}} \ldots \sum_{u_{k}=0}^{m_{k}} u_{1} N\left(m_{1}-u_{1}, \ldots, m_{k}-u_{k}\right) L\left(u_{1}, \ldots, u_{k}\right),
\end{aligned}
$$

where $L\left(u_{1}, \ldots, u_{k}\right)$ is defined by (2.2). This completes the proof of the lemma.

We note that equating the degrees in $x_{i}(i=2, \ldots, k)$ in $(2.8)$ yields only those companion formulae to $(2.1)$ obtainable by symmetry. 


\section{Further preliminaries}

Before applying Lemma 1, we require some further notation and preliminary results. For convenience, we shall assume that $\left(m_{1}, \ldots, m_{k}\right)(k \geqq 2)$ is totally positive.

Let $n, i$ be integers with $1 \leqq i \leqq n$. In what follows, we shall denote by $\sum_{n, i}^{\prime}$ a sum over all distinct selections (disregarding order) of $i$ elements, $j_{1}, \ldots, j_{i}$, out of the set $\{1,2, \ldots, n\}$. The remaining $n-i$ elements (in any order) will be denoted by $j_{i+1}, \ldots, j_{n}$.

We define $\Sigma_{t}$ and $\sum_{1}$ to be the sum over $r_{j_{t}}$ as $r_{j_{t}}$ takes integral values from 0 to $m_{j_{t}}-1$ and from 1 to $m_{j_{t}}-1$, respectively. Here, as usual, empty sums are taken to be zero. We denote the multiple sums $\Sigma_{1} \ldots \Sigma_{i}$ and $\sum_{1} \ldots \sum_{i}$ by $\Sigma^{(i)}$ and $\sum_{1}^{(i)}$, respectively. Write

$$
\begin{gathered}
Q_{0}=q^{\left(m_{1}+1\right) \ldots\left(m_{k}+1\right)} \\
Q_{i}=\sum_{k, i}^{\prime} q^{m_{J_{1}} \ldots m_{J_{i}}\left(m_{J_{i+1}}+1\right) \ldots\left(m_{j_{k}}+1\right)}, \quad(i=1, \ldots, k) .
\end{gathered}
$$

Then the function $N\left(m_{1}, \ldots, m_{k}\right)$ has the properties given in Lemma 2 below.

Lemma 2. We have

Moreover,

$$
(q-1) N\left(m_{1}, \ldots, m_{k}\right)=\sum_{i=0}^{k}(-1)^{i} Q_{i}
$$

and

$$
N\left(m_{1}, \ldots, m_{k}\right)=O\left(Q_{0}\right)
$$

$$
\begin{gathered}
N\left(m_{1}, \ldots, m_{k}\right)-q N\left(m_{1}, \ldots, m_{k}-1\right)=\left(1-q^{-n_{k}}\right) N\left(m_{1}, \ldots, m_{k}\right) \\
+\sum_{j=1}^{k-1} O\left(q^{m_{j} m_{k}\left(m_{j}+1\right)^{-1}\left(n_{k}+1\right)}\right),
\end{gathered}
$$

where

$$
n_{k}=\left\{\prod_{t=1}^{k-1}\left(m_{t}+1\right)\right\}-1,
$$

and the constants implied by the O-terms depend only on $q$.

Proof. In this proof we shall be considering only polynomials of degree at most $m_{i}$ in $x_{i}(i=1, \ldots, k)$. By the principal terms of a polynomial $f\left(x_{1}, \ldots, x_{k}\right)$ of degree $\left(m_{1}, \ldots, m_{k}\right)$, we shall mean those terms of $f\left(x_{1}, \ldots, x_{k}\right)$ which contain $x_{j}^{m_{j}}$ for at least one $j, 1 \leqq j \leqq k$. Thus, $N\left(m_{1}, \ldots, m_{k}\right)$ is the number of normalised polynomials which contain at least one principal term involving $x_{i}^{m_{i}}$ for each $i, i=1, \ldots, k$.

Now, for each $i$, with $0 \leqq i \leqq k$, the number of polynomials in which $x_{1}, \ldots, x_{i}$ (say) do not appear to the $m_{i}$ th power and in which the coefficients of principal terms not involving $x_{1}^{m_{1}}, \ldots, x_{i}^{m_{i}}$ may be zero is

$$
q^{m_{1} \ldots m_{t}\left(m_{t+1}+1\right) \ldots\left(m_{k}+1\right)}, \quad i=0, \ldots, k
$$


(with obvious conventions for $i=0$ or $k$ ). The sum, for fixed $i$, of all terms of the form (3.5) is $Q_{i}(i=0, \ldots, k)$.

Hence, for any $r$, with $0 \leqq r \leqq i$, the number of times a polynomial in which exactly $x_{1}, \ldots, x_{i}$ (say) do not appear to the powers $m_{1}, \ldots, m_{i}$, respectively, is counted in the number $Q_{r}$ is $\left(\begin{array}{c}i \\ i-r\end{array}\right)=\left(\begin{array}{l}i \\ r\end{array}\right)$ and so the number of times such a polynomial is counted in the sum

is

$$
S=\sum_{r=0}^{k}(-1)^{r} Q_{r}
$$

$$
\sum_{r=0}^{i}(-1)^{r}\left(\begin{array}{l}
i \\
r
\end{array}\right)= \begin{cases}1, & i=0, \\
0, & i=1, \ldots, k .\end{cases}
$$

Hence $S$ is exactly the number of polynomials of degree $\left(m_{1}, \ldots, m_{k}\right)$. Since the number of equivalence classes is $(q-1)^{-1} S$, result (3.1) follows.

(3.2) is a simple consequence of (3.1).

For (3.3), consider

$$
\begin{aligned}
& (q-1)\left[q^{-n_{k}} N\left(m_{1}, \ldots, m_{k}\right)-q N\left(m_{1}, \ldots, m_{k}-1\right)\right] \\
& =q^{-n_{k}}\left(q^{\left(m_{1}+1\right) \ldots\left(m_{k}+1\right)}-q^{m_{1}\left(m_{2}+1\right) \ldots\left(m_{k}+1\right)}-\ldots-q^{\left(m_{1}+1\right) \ldots\left(m_{k}-1+1\right) m_{k}}\right. \\
& + \text { terms of smaller order) } \\
& -q\left(q^{\left(m_{1}+1\right) \ldots\left(m_{k-1}+1\right) m_{k}}-q^{m_{1}\left(m_{2}+1\right) \ldots\left(m_{k-1}+1\right) m_{k}}-\ldots-q^{\left(m_{1}+1\right) \ldots\left(m_{k-1}+1\right)\left(m_{k}-1\right)}\right.
\end{aligned}
$$

+ terms of smaller order),

by (3.1). This, ignoring the terms of smaller order, equals

$$
\begin{aligned}
{\left[q^{\left(m_{1}+1\right) \ldots\left(m_{k-1}+1\right) m_{k}+1}\right.} & \left.-\ldots-q^{\left(m_{1}+1\right) \ldots\left(m_{k-1}+1\right)\left(m_{k}-1\right)+1}\right] \\
& -\left[q^{\left(m_{1}+1\right) \ldots\left(m_{k-1}+1\right) m_{k}+1}-\ldots-q^{\left(m_{1}+1\right) \ldots\left(m_{k-1}+1\right)\left(m_{k}-1\right)+1}\right] .
\end{aligned}
$$

The terms displayed cancel out in pairs, and the remaining terms give rise to the error terms shown in (3.3) (on dividing by $q-1$ ). The result (3.3) follows. This completes the proof of the lemma.

Note. For large $q$,

$$
N\left(m_{1}, \ldots, m_{k}\right)=O\left(q^{\left(m_{1}+1\right) \ldots\left(m_{k}+1\right)-1}\right) .
$$

In the present context $q$ is fixed.

From Lemma 1 and (3.2), we have

$$
L\left(m_{1}, \ldots, m_{k}\right) \leqq N\left(m_{1}, \ldots, m_{k}\right)=O\left(q^{\left(m_{1}+1\right) \ldots\left(m_{k}+1\right)}\right) .
$$

We shall use (3.6) later in the estimation of $L\left(m_{1}, \ldots, m_{k}\right)$.

Now, for fixed positive integers $m_{1}, \ldots, m_{k}$ and non-negative integers $r_{1}, \ldots, r_{k}$ write

$$
T\left(r_{1}, \ldots, r_{k}\right)=\left(r_{1}+1\right) \ldots\left(r_{k}+1\right)+\left(m_{1}-r_{1}+1\right) \ldots\left(m_{k}-r_{k}+1\right)
$$


so that, by (3.6),

$$
\begin{aligned}
L\left(r_{1}, \ldots, r_{k}\right) & N\left(m_{1}-r_{1}, \ldots, m_{k}-r_{k}\right) \\
& \leqq N\left(r_{1}, \ldots, r_{k}\right) N\left(m_{1}-r_{1}, \ldots, m_{k}-r_{k}\right)=O\left(q^{T\left(r_{1}, \ldots, r_{k}\right)}\right) .
\end{aligned}
$$

The following properties of $T\left(r_{1}, \ldots, r_{k}\right)$ are immediate; viz.

$$
T\left(r_{j_{1}}, \ldots, r_{j_{k}}\right)=T\left(r_{1}, \ldots, r_{k}\right),
$$

for any permutation $\left\{j_{1}, \ldots, j_{k}\right\}$ of $\{1, \ldots, k\}$, the $m_{i}$ 's being permuted in the same way, and

$$
T\left(m_{1}-r_{1}, \ldots, m_{k}-r_{k}\right)=T\left(r_{1}, \ldots, r_{k}\right) .
$$

We shall require upper bounds for terms of the form (3.7); we obtain these in Lemmas 3 and 4 below.

Lemma 3. Let $m_{1}, \ldots, m_{k}, u$ be fixed positive integers such that $1 \leqq u \leqq k-1$, $m_{t} \geqq 2(u<t \leqq k)$. Suppose also that $s_{1}, \ldots, s_{k}$ are integers satisfying

$$
\left.\begin{array}{ll}
0 \leqq s_{t} \leqq m_{t}, & (1 \leqq t \leqq u), \\
1 \leqq s_{t} \leqq m_{t}-1, & (u<t \leqq k) ;
\end{array}\right\}
$$

then

$$
T\left(s_{1}, \ldots, s_{k}\right) \leqq T\left(m_{1}, \ldots, m_{u}, m_{u+1}-1, \ldots, m_{k}-1\right) .
$$

Proof. To prove (3.11), we observe that if $2 s_{t} \geqq m_{t}, t=1, \ldots, k$, then, for each $j$ with $1 \leqq j \leqq k$,

$$
\frac{\partial T}{\partial s_{j}}\left(s_{1}, \ldots, s_{k}\right)=\frac{1}{s_{j}+1} \prod_{t=1}^{k}\left(s_{t}+1\right)-\frac{1}{m_{j}-s_{j}+1} \prod_{t=1}^{k}\left(m_{t}-s_{t}+1\right) \geqq 0
$$

at the point $\left(s_{1}, \ldots, s_{k}\right)$. Thus $T$ is increasing with respect to $s_{j}$ for each $j$, $1 \leqq j \leqq k$. Hence for this case (3.11) holds.

We now show that for general $s_{1}, \ldots, s_{k}$ satisfying (3.10), there exist integers $s_{1}^{\prime}, \ldots, s_{k}^{\prime}$ also satisfying (3.10) with, in addition, $2 s_{t}^{\prime} \geqq m_{t}(t=1, \ldots, k)$ and

$$
T\left(s_{1}, \ldots, s_{k}\right) \leqq T\left(s_{1}^{\prime}, \ldots, s_{k}^{\prime}\right) .
$$

Thus, by the first part of the proof applied to $T\left(s_{1}^{\prime}, \ldots, s_{k}^{\prime}\right)$ and $(3.12),(3.11)$ is proved.

Let $v$ be the number of the $s_{t}(t=1, \ldots, k)$ such that $2 s_{t} \geqq m_{t}$ and so $0 \leqq v \leqq k$. We have already discussed the case $v=k$ and the case $v=0$ reduces to it on application of (3.9) and noting that (3.10) remains valid with $s_{t}$ replaced by $m_{t}-s_{t}(t=1, \ldots, k)$. Suppose now that $v \neq 0, k$ and let $s_{j_{1}}, \ldots, s_{j_{k}}$ be a rearrangement of $s_{1}, \ldots, s_{k}$ such that $2 s_{j_{1}} \geqq m_{j_{i}}(1 \leqq i \leqq v)$ and $2 s_{j_{i}}<m_{j_{i}}(v+1 \leqq i \leqq k)$. Now, if $s_{t}$ satisfies one of the pair of inequalities in (3.10), $m_{t}-s_{t}$ satisfies the same inequality. Further $2\left(m_{j_{i}}-s_{j_{i}}\right) \geqq m_{j_{i}}$, 
$v+1 \leqq i \leqq k$. Hence

$$
\begin{aligned}
& T\left(s_{j_{t}}, \ldots, s_{j_{v}}, m_{j_{v+1}}-s_{j_{v+1}}, \ldots, m_{j_{k}}-s_{j_{k}}\right)-T\left(s_{j_{1}}, \ldots, s_{j_{k}}\right) \\
& \quad=\left\{\prod_{i=1}^{0}\left(s_{j_{i}}+1\right)-\prod_{i=1}^{0}\left(m_{j_{t}}-s_{j_{t}}+1\right)\right\}\left\{\prod_{i=v+1}^{k}\left(m_{j_{t}}-s_{j_{t}}+1\right)-\prod_{i=v+1}^{k}\left(s_{j_{t}}+1\right)\right\} \\
& \quad \geqq 0 .
\end{aligned}
$$

Whence (3.12) holds with

$$
s_{j_{t}}^{\prime}=\left\{\begin{array}{l}
s_{j_{i}}, 1 \leqq i \leqq v \\
m_{j_{i}}-s_{j_{i}}, v+1 \leqq i \leqq k,
\end{array}\right.
$$

after using the permutation rule (3.8). This completes the proof. Then

Lemma 4. Let $m_{1}, \ldots, m_{k}$, $u$ be fixed positive integers with $1 \leqq u \leqq k-1$.

$$
\begin{aligned}
& T\left(m_{1}, \ldots, m_{u}, 0, \ldots, 0\right) \\
& \quad \leqq \max \left\{T\left(m_{1}, \ldots, m_{u}, m_{u+1}-1, \ldots, m_{k}-1\right),\right. \\
& \left.\quad T\left(m_{1}-1, \ldots, m_{u}-1, m_{u+1}, \ldots, m_{k}\right)\right\} .
\end{aligned}
$$

Proof. (i) Suppose first that $2 u \geqq k$. Then

$$
\begin{aligned}
T\left(m_{1}, \ldots,\right. & \left.m_{u}, m_{u+1}-1, \ldots, m_{k}-1\right)-T\left(m_{1}, \ldots, m_{u}, 0, \ldots, 0\right) \\
& =\left(m_{1}+1\right) \ldots\left(m_{u}+1\right)\left(m_{u+1} \ldots m_{k}-1\right)-\left[\left(m_{u+1}+1\right) \ldots\left(m_{k}+1\right)-2^{k-u}\right] \\
& \geqq 2^{u}\left(m_{u+1} \ldots m_{k}-1\right)-2^{k-u}\left(m_{u+1} \ldots m_{k}-1\right) \\
& \geqq 0 .
\end{aligned}
$$

(ii) Suppose now that $2 u<k$. By (3.9), (3.8)

$$
\begin{aligned}
T\left(m_{1}, \ldots, m_{u}, 0, \ldots, 0\right) & =T\left(0, \ldots, 0, m_{u+1}, \ldots, m_{k}\right) \\
& =T\left(m_{u+1}, \ldots, m_{k}, 0, \ldots, 0\right) \\
& \leqq T\left(m_{u+1}, \ldots, m_{k}, m_{1}-1, \ldots, m_{u}-1\right),
\end{aligned}
$$

by (i), since $2(k-u)>k$.

This completes the proof.

Evidently,

$T\left(m_{1}, \ldots, m_{u}, m_{u+1}-1, \ldots, m_{k}-1\right)=\left(m_{1}+1\right) \ldots\left(m_{u}+1\right) m_{u+1} \ldots m_{k}+O(1)$.

In fact, the constant in (3.14) is $2^{k-u}$.

4. Estimation of $M\left(m_{1}, \ldots, m_{k}\right)$

It may be verified by direct substitution that we can rewrite (2.2) as

$$
M\left(m_{1}, \ldots, m_{k}\right)=\sum_{j \mid\left(m_{1}, \ldots, m_{k}\right)} \frac{\mu(j)}{j} L\left(\frac{m_{1}}{j}, \ldots, \frac{m_{k}}{j}\right),
$$

where $\mu(j)$ is the Möbius function. 
Using the results of $\S \S 2-3$, we obtain an estimate for $L\left(m_{1}, \ldots, m_{k}\right)$ and invoke (4.1) to deduce the corresponding estimate for $M\left(m_{1}, \ldots, m_{k}\right)$. We restrict ourselves again, without loss of generality, to the case $\left(m_{1}, \ldots, m_{k}\right)$ totally positive.

We recall, from Lemma 1,

$$
\begin{aligned}
m_{1} N\left(m_{1}, \ldots, m_{k}\right) & =\sum_{r_{1}=0}^{m_{1}} \ldots \sum_{r_{k}=0}^{m_{k}} r_{1} L\left(r_{1}, \ldots, r_{k}\right) N\left(m_{1}-r_{1}, \ldots, m_{k}+r_{k}\right) \\
& =m_{1} \sum_{r_{k}=0}^{m_{k}} L\left(m_{1}, \ldots, m_{k-1}, r_{k}\right) N\left(0, \ldots, 0, m_{k}-r_{k}\right)+m_{1} E,
\end{aligned}
$$

where, since $r_{1} / m_{1} \leqq 1$, we have

$$
\begin{aligned}
E \leqq \sum_{i=1}^{k-1} \sum_{k=1, i}^{\prime} \sum^{(i)} \sum_{r_{k}=0}^{m_{k}}\left\{L \left(r_{j_{1}}, \ldots,\right.\right. & \left.r_{j_{i}}, m_{j_{t+1}}, \ldots, m_{j_{k-1}}, r_{k}\right) \\
& \left.\times N\left(m_{j_{1}}-r_{j_{1}}, \ldots, m_{j_{t}}-r_{j_{t}}, 0, \ldots, 0, m_{k}-r_{k}\right)\right\} .
\end{aligned}
$$

Using (3.7), we obtain

$$
E=\sum_{i=1}^{k-1} \sum_{k=1, i}^{\prime} \sum^{(i)} \sum_{r_{k}=0}^{m_{k}} O\left\{q^{T\left(r_{j_{1}}, \ldots, r_{f_{t}}, m_{j_{i+1}}, \ldots, m_{j_{k-1}}, r_{k}\right)}\right\}
$$

where the implied constants depend only on $q$. We divide the summation of (4.3) into two cases:

(i) $r_{j_{t}}=0(t=1, \ldots, i)$. These terms are restated in (4.5) below. Since the summand in (2.1) contains the factor $r_{1}$ we can neglect terms in which $r_{1}$ takes the value zero in (4.3). This justifies the omission of the term

$$
\sum_{r_{k}=0}^{m_{k}} O\left(q^{T\left(0, \ldots, 0, m_{k}\right)}\right)
$$

from the second multiple sum of (4.5).

(ii) $r_{j_{t}} \geqq 1$, for some $t$ with $1 \leqq t \leqq i$. On application of Lemma 3 , these terms give rise to a sum

$$
\sum_{i=1}^{k-1} \sum_{k=1, i}^{\prime} \sum_{s=1}^{i-1} \sum_{i, s}^{\prime} \sum_{1}^{\left(j_{s}\right)} \sum_{r_{k}=0}^{m_{k}} O\left\{q^{T\left(m_{(1)}-1, \ldots, m_{(s)}-1, m_{(s+1)}, \ldots, m_{k}\right)}\right\},
$$

where the subscript $(t)$ in $m_{(t)}$ denotes $j_{j_{t}}$. The sum (4.4) can be rewritten to give the first multiple sum in the expression

$$
\begin{aligned}
E=\sum_{i=1}^{k-1} \alpha_{i} \sum_{k=1, i}^{\prime} \sum_{1}^{(i)} \sum_{r_{k}=0}^{m_{k}} O\left\{q^{T\left(m_{j_{1}}-1, \ldots, m_{j_{k}}-1, m_{j_{i+1}}, \ldots, m_{k}\right)}\right\} & \\
& +\sum_{i=1}^{k-2} \sum_{k=1, i}^{\prime} \sum_{r_{k}=0}^{m_{k}} O\left\{q^{T\left(0, \ldots, 0, m_{j_{i+1}}, \ldots, m_{k}\right)}\right\},
\end{aligned}
$$


where, for $1 \leqq i \leqq k-1$, we have

$$
\begin{aligned}
\alpha_{i} & =1+\left(\begin{array}{c}
k-1-i \\
1
\end{array}\right)+\left(\begin{array}{c}
k-1-i \\
2
\end{array}\right)+\ldots+\left(\begin{array}{l}
k-1-i \\
k-1-i
\end{array}\right) \\
& =2^{k-1-i} .
\end{aligned}
$$

Application of Lemma 4 and (3.14) to (4.5) yields

$$
E=\sum_{i=1}^{k-1} \sum_{k=1, i}^{\prime} O\left(m_{j_{1}} \ldots m_{j_{i}} q^{m_{j_{1}} \ldots m_{j_{i}}\left(m_{j_{i}+1}+1\right) \ldots\left(m_{k}+1\right)}\right)
$$

where the constants implied by the $O$-terms are of the form $\beta_{i} B$, where $B$ depends only on $q$ and, for $1 \leqq i \leqq k-1$,

$$
\begin{aligned}
\beta_{i} & = \begin{cases}\left(\alpha_{i}+1\right) q^{2 i}, & 2 i \leqq k \\
\alpha_{i} q^{2 i}+q^{2^{k-i}}, & 2 i>k\end{cases} \\
& \leqq\left(2^{k-1-i}+1\right) q^{2^{i}} .
\end{aligned}
$$

For fixed $k$, we can neglect many of the terms in (4.6) and, in fact, obtain

$$
E=\sum_{i=1}^{[k-1} O\left(m_{i} m_{k} q^{m_{l}\left(m_{i}+1\right)^{-1}\left(n_{k}+1\right)\left(m_{k}+1\right)}\right)
$$

where $n_{k}$ is given by (3.4) and the implied constants depend only on $q$ and $k$.

Accordingly, by (4.2) and (4.7),

$$
N\left(m_{1}, \ldots, m_{k}\right)=\sum_{r_{k}=0}^{m_{k}} q^{m_{k}-r_{k}} L\left(m_{1}, \ldots, m_{k-1}, r_{k}\right)+\sum_{i=1}^{k-1} O\left(c_{i}\right),
$$

where, for $1 \leqq i \leqq k-1$,

$$
c_{i}=m_{i} m_{k} q^{m_{i}\left(m_{i}+1\right)-1\left(n_{k}+1\right)\left(m_{k}+1\right)}
$$

and the constants implied by the $O$-terms depend only on $q$ and $k$. It follows from (4.8) that, if $m_{k} \geqq 2$,

$$
N\left(m_{1}, \ldots, m_{k}\right)-q N\left(m_{1}, \ldots, m_{k}-1\right)=L\left(m_{1}, \ldots, m_{k}\right)+\sum_{i=1}^{k-1} O\left(c_{i}\right) .
$$

Comparing (3.3) and (4.9) we obtain

$$
L\left(m_{1}, \ldots, m_{k}\right)=\left(1-q^{-n_{k}}\right) N\left(m_{1}, \ldots, m_{k}\right)+\sum_{i=0}^{k-1} O\left(c_{i}\right) .
$$

Now, if $\left(m_{1}, \ldots, m_{k}\right)=1$, i.e. if $m_{1}, \ldots, m_{k}$ are relatively prime, it follows from $(4,1)$ that

$$
M\left(m_{1}, \ldots, m_{k}\right)=L\left(m_{1}, \ldots, m_{k}\right) .
$$

In particular (4.11) holds when $m_{j}=1$ for some $j, 1 \leqq j \leqq k$. If $m_{j} \geqq 2$, $j=1, \ldots, k$, we have from (4.1) and (3.6)

$$
\begin{aligned}
M\left(m_{1}, \ldots, m_{k}\right) & =L\left(m_{1}, \ldots, m_{k}\right)+O\left(q^{\left(m_{1}+2\right) \ldots\left(m_{k}+2\right) / 2^{k}}\right) \\
& =L\left(m_{1}, \ldots, m_{k}\right)+O\left(q^{m_{1} \ldots m_{k}}\right) .
\end{aligned}
$$


Thus, combining (4.11) and (4.12), (4.12) holds for all totally positive $\left(m_{1}, \ldots, m_{k}\right)$. Here again the implied constant depends only on $q$.

From (4.10) and (4.12), we obtain an expression for $M\left(m_{1}, \ldots, m_{k}\right)$ which is the main result of the following theorem.

Theorem 1. Let $m_{1}, \ldots, m_{k}$ be positive integers with $k \geqq 2$. Then the number $M\left(m_{1}, \ldots, m_{k}\right)$ of normalised irreducible polynomials of degree $\left(m_{1}, \ldots, m_{k}\right)$ in $k$ indeterminates with coefficients in $G F[q]$ satisfies

$$
\begin{aligned}
M\left(m_{1}, \ldots, m_{k}\right)=\left(1-q^{-n_{k}}\right) & N\left(m_{1}, \ldots, m_{k}\right) \\
& +\sum_{i=1}^{k-1} O\left(m_{i} m_{k} q^{m_{i}\left(m_{i}+1\right)-1\left(n_{k}+1\right)\left(m_{k}+1\right)}\right),
\end{aligned}
$$

where $N\left(m_{1}, \ldots, m_{k}\right)$ is the total number of normalised polynomials of degree $\left(m_{1}, \ldots, m_{k}\right), n_{k}$ is given by (3.4) and the constants implied by the O-terms depend only on the field (i.e. on $q$ ) and on $k$.

In particular, for fixed $m_{1}, \ldots, m_{k-1}$, numbered so that

$$
\begin{gathered}
m_{k-1}=\max _{1 \leqq i \leqq k-1} m_{i}, \\
M\left(m_{1}, \ldots, m_{k}\right)=\left(1-q^{-n_{k}}\right) N\left(m_{1}, \ldots, m_{k}\right)+O\left(m_{k} q^{\left(m_{1}+1\right) \ldots\left(m_{k-2}+1\right) m_{k-1} m_{k}}\right),
\end{gathered}
$$

where the implied constant is independent of $m_{k}$; and

$$
M\left(m_{1}, \ldots, m_{k}\right) \sim\left(1-q^{-n_{k}}\right) N\left(m_{1}, \ldots, m_{k}\right) \text { as } m_{k} \rightarrow \infty .
$$

Further, for $k \geqq 3$ and fixed $m_{1}, \ldots, m_{k-2}$,

$$
M\left(m_{1}, \ldots, m_{k}\right) \sim N\left(m_{1}, \ldots, m_{k}\right) \text { as } m_{k-1}, m_{k} \rightarrow \infty .
$$

Proof. We have already proved (4.13). Statements (4.14) and (4.15) are immediate from (4.13). Also (4.16) follows from (4.15) on observing that, for fixed $m_{1}, \ldots, m_{k-2}$,

$$
q^{-n_{k}} N\left(m_{1}, \ldots, m_{k}\right)=o\left(N\left(m_{1}, \ldots, m_{k}\right)\right) \text { as } m_{k-1}, m_{k} \rightarrow \infty .
$$

This completes the proof.

We note that, by (4.16), we can say that, if $2 \leqq j \leqq k$,

$$
M\left(m_{1}, \ldots, m_{k}\right) \sim N\left(m_{1}, \ldots, m_{k}\right) \quad(k \geqq 2)
$$

holds as any $j$ of $m_{1}, \ldots, m_{k} \rightarrow \infty$.

We investigate (4.14) and (4.16) in more detail in $\S 5$ below.

\section{Improvement of (4.14) and (4.16)}

We consider first the estimation of $M\left(m_{1}, \ldots, m_{k}\right)$ in which $m_{1}, \ldots, m_{k-1}$ are fixed. In this case we can improve (4.14) except when $k=2$ and $m_{1}=2$ and when $k=3$ and $m_{1}=m_{2}=1$.

We can calculate $M(2, n)$ and $M(1,1, n)$, where $n \geqq 1$, directly using Lemma 1 and (4.1) (see $\S 6$ ). When expressed in the form of (4.14), we obtain

$$
M(2, n)=\left(1-q^{-2}\right) N(2, n)-\frac{1}{2} q^{2}\left(1-q^{-2}\right)^{2} n q^{2 n}+O\left(q^{2 n}\right),
$$


and

$$
M(1,1, n)=\left(1-q^{-3}\right) N(1,1, n)-q^{2}\left(1-q^{-2}\right)^{2} n q^{2 n}+O\left(q^{2 n}\right),
$$

both expressions being valid for large $n$. We see that in these cases (4.14) is "best possible".

Define the positive integer $R$ by

$$
\left.\begin{array}{l}
R=1, \text { if } k=2, \\
R=\left(m_{1}+1\right) \ldots\left(m_{k-2}+1\right), \text { if } k \geqq 3 .
\end{array}\right\}
$$

Then apart from the cases already considered we may replace (4.14) by the improvement contained in the following theorem.

Theorem 2. Let $m_{1}, \ldots, m_{k-1}(k \geqq 2)$ be fixed positive integers numbered so that $m_{1} \leqq m_{2} \leqq \ldots \leqq m_{k-1}$. Then, in the notation of Theorem 1, $M\left(m_{1}, \ldots, m_{k}\right)$ satisfies, with the exceptions of $M\left(2, m_{2}\right)$ and $M\left(1,1, m_{3}\right)$,

$$
M\left(m_{1}, \ldots, m_{k}\right)=\left(1-q^{-n_{k}}\right) N\left(m_{1}, \ldots, m_{k}\right)+O\left(q^{R m_{k}-1 m_{k}}\right)
$$

for large $m_{k}$, where $R$ is given by (5.1) and the implied constant is independent of $m_{k}$. Further, (5.2) is the "best possible" estimate for $M\left(m_{1}, \ldots, m_{k}\right)$, i.e., there exists a positive number $K$, independent of $m_{k}$, such that, for fixed $m_{1}, \ldots, m_{k-1}$,

$$
\mid M\left(m_{1}, \ldots, m_{k}\right)-\left(1-q^{-n_{k}}\right) N\left(m_{1}, \ldots, m_{k} \mid>K q^{R m_{k-1} m_{k}}\right.
$$

holds.

Proof. In this proof let $\Delta$ denote the difference

$$
N\left(m_{1}, \ldots, m_{k}\right)-q N\left(m_{1}, \ldots, m_{k}-1\right)
$$

We require the following consequences of (3.1), valid for large $m_{k}$. We have

$$
\begin{aligned}
& N\left(m_{1}, \ldots, m_{k-2}, m_{k-1}-1, m_{k}\right)=(q-1)^{-1}\left(q^{R m_{k-1}}-1\right) q^{R m_{k-1} m_{k}} \\
& +O\left(q^{R\left(m_{k}-1-1\right) m_{k}}\right),
\end{aligned}
$$

$\Delta=\left(1-q^{-n_{k}}\right) N\left(m_{1}, \ldots, m_{k}\right)$

$$
+\left(1-q^{-1}\right)^{-1}\left(1-q^{-R}\right)\left(1-q^{-R m_{k-1}}\right) q^{R m_{k-1} m_{k}}+o\left(q^{R m_{k-1} m_{k}}\right) .
$$

We verify (5.4) and (5.5) as follows. 'Firstly, by (3.1), we have

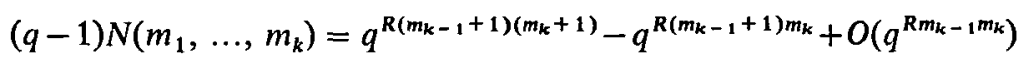

$$
\begin{aligned}
& =\left(q^{R\left(m_{k}-1+1\right)}-1\right) q^{R\left(m_{k}-1+1\right) m_{k}}+O\left(q^{R m_{k}-1 m_{k}}\right) .
\end{aligned}
$$

This gives (5.4) (on replacing $m_{k-1}$ by $m_{k-1}-1$ ). To prove (5.5), consider the expression $E_{1}$, where

Then

$$
E_{1}=\Delta-\left(1-q^{-n_{k}}\right) N\left(m_{1}, \ldots, m_{k}\right) \text {. }
$$

$$
E_{1}=q^{-n_{k}} N\left(m_{1}, \ldots, m_{k}\right)-q N\left(m_{1}, \ldots, m_{k}-1\right) \text {. }
$$


Thus, by (3.1),

$$
\begin{aligned}
& (q-1) E_{1}=q^{-R\left(m_{k-1}+1\right)+1}\left[q^{R\left(m_{k-1}+1\right)\left(m_{k}+1\right)}-q^{R\left(m_{k-1}+1\right) m_{k}}-q^{R m_{k}-1\left(m_{k}+1\right)}\right. \\
& \left.+q^{R m_{k}-1 m_{k}}+o\left(q^{R m_{k}-1 m_{k}}\right)\right] \\
& -q\left[q^{R\left(m_{k}-1+1\right) m_{k}}-q^{R\left(m_{k-1}+1\right)\left(m_{k}-1\right)}-q^{R m_{k-1} m_{k}}+q^{R m_{k}-1\left(m_{k}-1\right)}+o\left(q^{R m_{k}-1 m_{k}}\right)\right] .
\end{aligned}
$$

The first two terms in the first large bracket cancel with the corresponding terms in the second large bracket as in the proof of Lemma 2. Hence, ignoring the $o$-terms, we have

$$
\begin{aligned}
(q-1) E_{1} & =q^{R m_{k}-1 m_{k}+1}\left[-q^{-R}+q^{-R\left(m_{k-1}+1\right)}+1-q^{-R m_{k}-1}\right] \\
& =q^{R m_{k-1} m_{k}+1}\left(1-q^{-R}\right)\left(1-q^{-R m_{k}-1}\right),
\end{aligned}
$$

which yields (5.5) on division by $q-1$.

Now, from (4.2), $\Delta$ is also given by

$$
\begin{gathered}
\Delta=L\left(m_{1}, \ldots, m_{k}\right) \\
+\sum_{i=1}^{k-1} \sum_{k=1, i}^{\prime} \sum^{(i)} \sum_{r_{k}=0}^{m_{k}}\left[\left\{\frac{r_{1}}{m_{1}} L\left(r_{j_{1}}, \ldots, r_{j_{i}}, m_{j_{t+1}}, \ldots, m_{j_{k-1}}, r_{k}\right)\right\}\right] \\
\times\left\{N\left(m_{j_{1}}-r_{j_{1}}, \ldots, m_{j_{i}}-r_{j_{t}}, 0, \ldots, 0, m_{k}-r_{k}\right)\right. \\
\left.\quad-q N\left(m_{j_{1}}-r_{j_{1}}, \ldots, m_{j_{i}}-r_{j_{i}}, 0, \ldots, 0, m_{k}-r_{k}-1\right)\right\} \\
+\sum_{i=1}^{k-1} \sum_{k=1, i}^{\prime} \sum^{(i)}\left[\left\{\frac{r_{1}}{m_{1}} L\left(r_{j_{1}}, \ldots, r_{j_{i}}, m_{j_{i+1}}, \ldots, m_{j_{k-1}}, m_{k}\right)\right\}\right. \\
\left.\times\left\{N\left(m_{j_{1}}-r_{j_{1}}, \ldots, m_{j_{i}}-r_{j_{i}}, 0, \ldots, 0\right)\right\}\right] .
\end{gathered}
$$

Let $\alpha$ be the number of $m_{t}, 1 \leqq t \leqq k-1$, such that $m_{t}=m_{k-1}$. Also define the integer $t_{k}$ by

$$
t_{k}=R m_{k-1}-1 \text {. }
$$

Then a fairly long, complicated argument, which we shall omit, shows that, except when $k=2$ and $m_{1}=2$ and when $k=3$ and $m_{1}=m_{2}=1$, from (5.6), it follows that

$$
\begin{aligned}
\Delta & =L\left(m_{1}, \ldots, m_{k}\right) \\
& +\beta\left(1-q^{-1}\right) \sum_{r_{k}=0}^{m_{k}-1} L\left(m_{1}, \ldots, m_{k-2}, m_{k-1}-1, r_{k}\right) N\left(0, \ldots, 0,1, m_{k}-r_{k}\right) \\
& +(\alpha-\beta)\left(1-q^{-t_{k}}\right) \sum_{r_{k}=0}^{m_{k}-1} L\left(0, \ldots, 0,1, m_{k}-r_{k}\right) N\left(m_{1}, \ldots, m_{k-2}, m_{k-1}-1, r_{k}\right) \\
& +\beta L\left(m_{1}, \ldots, m_{k-1}-1, m_{k}\right) N(0, \ldots, 0,1,0) \\
& +(\alpha-\beta)\left(1-q^{-t_{k}}\right) L(0, \ldots, 0,1,0) N\left(m_{1}, \ldots, m_{k-1}-1, m_{k}\right)+o\left(q^{R m_{k-1} m_{k}}\right),
\end{aligned}
$$


where

$$
\beta=\left\{\begin{array}{l}
\alpha-\frac{1}{m_{1}}, \text { if } \alpha=k-1 \\
\alpha, \text { otherwise. }
\end{array}\right.
$$

By (4.10), (2.14), (5.5) and (5.7) we have, with the same exceptions,

$$
\begin{aligned}
& M\left(m_{1}, \ldots, m_{k}\right)=\left(1-q^{-n_{k}}\right) N\left(m_{1}, \ldots, m_{k}\right) \\
& -\alpha\left(1-q^{-1}\right)\left(1-q^{-t_{k}}\right) \sum_{r_{k}=0}^{m_{k}-1} N\left(m_{1}, \ldots, m_{k-2}, m_{k-1}-1, r_{k}\right) N\left(0, \ldots, 0,1, m_{k}-r_{k}\right) \\
& -\alpha\left(1-q^{-t_{k}}\right) N\left(m_{1}, \ldots, m_{k-2}, m_{k-1}-1, m_{k}\right) N(0, \ldots, 0,1,0) \\
& +\left(1-q^{-1}\right)^{-1}\left(1-q^{-R}\right)\left(1-q^{-R m_{k-1}}\right) q^{R m_{k-1} m_{k}}+o\left(q^{R m_{k-1} m_{k}}\right) .
\end{aligned}
$$

For the proof of (5.2), we use (5.8) in the weaker form

$$
\begin{aligned}
M\left(m_{1}, \ldots, m_{k}\right)-\left(1-q^{-n_{k}}\right) & N\left(m_{1}, \ldots, m_{k}\right) \\
\quad= & \sum_{r_{k}=0}^{m_{k}-1} O\left(q^{T\left(m_{1}, \ldots, m_{k}-2, m_{k-1}-1, r_{k}\right)}\right)+O\left(q^{R m_{k}-1 m_{k}}\right) \\
= & O\left(q^{2 m_{k}}\right) \sum_{r_{k}=0}^{m_{k}-1} q^{r_{k}\left(R m_{k-1}-2\right)}+O\left(q^{R m_{k-1} m_{k}}\right),
\end{aligned}
$$

where the implied constants are independent of $m_{k}$. Now $R m_{k-1}-2=0$ if and only if either $R=1, k=2$ and $m_{k-1}=2$ or $R=2, k=3$ and $m_{1}=m_{2}=1$. These are the special cases already considered and excluded from this discussion. Otherwise, R.H.S. of (5.9) is

$$
\begin{aligned}
& O\left(q^{2 m_{k}+\left(m_{k}-1\right)\left(R m_{k}-1-2\right)}\right)+O\left(q^{R m_{k}-1 m_{k}}\right) \\
= & O\left(q^{R m_{k}-1 m_{k}}\right),
\end{aligned}
$$

where the implied constant is independent of $m_{k}$. This proves (5.2).

To prove (5.3), we again consider (5.8). By (5.4), we have

$$
\begin{aligned}
& N(0, \ldots, 0,1,0) N\left(m_{1}, \ldots, m_{k-2}, m_{k-1}-1, m_{k}\right)=\left(1-q^{-1}\right)^{-1}\left(q^{t_{k}+1}-1\right) q^{R m_{k-1} m_{k}} \\
& +o\left(q^{R m_{k-1} m_{k}}\right) .
\end{aligned}
$$

$$
\Delta_{1}=M\left(m_{1}, \ldots, m_{k}\right)-\left(1-q^{-n_{k}}\right) N\left(m_{1}, \ldots, m_{k}\right),
$$

it follows from (5.8) and (5.10) that

$$
\begin{aligned}
\Delta_{1} \leqq\left[\left(1-q^{-1}\right)^{-1}(1-\right. & \left.q^{-R}\right)\left(1-q^{-\left(t_{k}+1\right)}\right) \\
& \left.-\alpha\left(1-q^{-1}\right)^{-1}\left(1-q^{-t_{k}}\right)\left(q^{t_{k}+1}-1\right)\right] q^{R m_{k}-1 m_{k}}+o\left(q^{R m_{k}-1 m_{k}}\right) \\
& =-K q^{R m_{k-1} m_{k}}+o\left(q^{R m_{k}-1 m_{k}}\right), \text { say. }
\end{aligned}
$$

Now, $-K$ is negative except when $t_{k}=0$, i.e., when $k=2$ and $m_{1}=1$. 
Hence, except in the case $M\left(1, m_{2}\right)$, we have, for large $m_{k}$,

$$
\left|\Delta_{1}\right| \geqq K q^{R m_{k}-1 m_{k}},
$$

where $K$ is positive and independent of $m_{k}$. Thus (5.3) is proved except, possibly, for $M\left(1, m_{2}\right)$. However, in this case, (5.3) can be verified directly. In fact,

$$
M(1, \mathrm{n})=\left(1-q^{-1}\right) N(1, n)+\left(1-q^{-1}\right) q^{n} .
$$

This completes the proof of Theorem 2.

From the results of $\S 5$, it also follows that

$$
M\left(m_{1}, \ldots, m_{k}\right)<\left(1-q^{-n_{k}}\right) N\left(m_{1}, \ldots, m_{k}\right),
$$

for large $m_{k}$, except when $k=2$ and $m_{1}=1$.

Suppose now that $k \geqq 3$ and that $m_{1}, \ldots, m_{k-2}$ are fixed, positive integers. In this case, Theorem 1 yields, for large $m_{k-1}$ and $m_{k}$,

$$
\begin{array}{ll}
\begin{array}{ll}
M\left(m_{1}, \ldots, m_{k}\right)=N\left(m_{1}, \ldots, m_{k}\right)-\left[\left(1-q^{-1}\right)^{-1}\right. & +o(1)] q^{R\left(m_{k}-1+1\right) m_{k}} \\
& +O\left(m_{k-1} m_{k} q^{R m_{k-1}\left(m_{k}+1\right)}\right),
\end{array} \\
\text { since, by (3.1), } &
\end{array}
$$

$$
q^{-n_{k}} N\left(m_{1}, \ldots, m_{k}\right)=\left(1-q^{-1}\right)^{-1} q^{R\left(m_{k-1}+1\right) m_{k}}+o\left(q^{R\left(m_{k}-1+1\right) m_{k}}\right) .
$$

Considerations of symmetry suggest that (5.12) can be improved to give the result of our final theorem.

Theorem 3. Let $m_{1}, \ldots, m_{k-2}$, where $k \geqq 3$, be fixed, positive integers. Then, in the notation of Theorem 1 ,

$$
\begin{aligned}
M\left(m_{1}, \ldots, m_{k}\right)=N\left(m_{1}, \ldots, m_{k}\right)-\left[\left(1-q^{-1}\right)^{-1}+o(1)\right] & \left(q^{R\left(m_{k}-1+1\right) m_{k}}+q^{R m_{k-1}\left(m_{k}+1\right)}\right)
\end{aligned}
$$

holds for large $m_{k-1}$ and $m_{k}$, where $R$ is determined by (5.1). In particular (4.16) holds.

Proof. The result follows on letting $m_{k-1}$ become large in (5.8) and using (5.13) and Lemma 2. By the choice of $m_{k-1}$ in Theorem 2 as $\max m_{i}$, $1 \leqq i \leqq k-1$, we can see that (5.8) is valid for large $m_{k-1}$ and $m_{k}$ (i.e., provided $\left.m_{k-1}>m_{i}, 1 \leqq i \leqq k-2\right)$. Also, we can take $\alpha=1$ in (5.8). In the right hand side of (5.8), apart from the first term, the only term which is not

is, by Lemma 2,

$$
o\left(q^{R m_{k}-1\left(m_{k}+1\right)}\right)
$$

$$
\left(1-q^{-t_{k}}\right) N\left(m_{1}, \ldots, m_{k-2}, m_{k-1}-1, m_{k}\right) N(0, \ldots, 0,1,0),
$$

which equals

$$
\left(1-q^{-1}\right)^{-1} q^{R m_{k-1}\left(m_{k}+1\right)}+o\left(q^{R m_{k-1}\left(m_{k}+1\right)}\right),
$$

as $m_{k-1}$ and $m_{k} \rightarrow \infty$. The proof is complete.

Note. Theorem 3 has been given for $k \geqq 3$ to make its statement easier. 
In fact, the same proof for $k=2$ shows that

$$
M(m, n)=N(m, n)-\left[\left(1-q^{-1}\right)^{-1}+o(1)\right]\left(q^{m}+q^{n}\right) q^{m n}
$$

holds as $m$ and $n$ tend to infinity.

\section{Examples}

We now list explicit values of $M\left(m_{1}, \ldots, m_{k}\right)$ for the simplest cases in which $k=2$ or 3 . They are obtained by calculating $L\left(m_{1}, \ldots, m_{k}\right)$ from (5.6), using previously calculated values of the $L$-function and (3.1), and then applying (4.1).

We obtain, in turn, for $n \geqq 1$,

$$
M(1, n)=\left(q^{2}-1\right) q^{2 n-1} ;
$$

$M(2, n)=\left(q^{2}-1\right)\left\{\left(q^{2}+q+1\right) q^{3 n-2}-\frac{1}{2}\left(\left(q^{2}-1\right) n+(q+1)^{2}\right) q^{2 n-2}-\frac{1}{2} \delta q^{n-1}\right\}$,

where $\delta$ is 1 or 0 according as $n$ is even or odd,

$$
\begin{aligned}
& M(3, n)=\left(q^{2}-1\right)\left\{\left(q^{2}+1\right)\left(q^{2}+q+1\right) q^{4 n-3}-(q+1)^{2}\left(q^{2}+q+1\right) q^{3 n-3}\right. \\
& \left.+\frac{1}{6}\left[\left(q^{2}-1\right)^{2} n^{2}+3(q-1)(q+1)^{3} n+2\left(q^{2}+q+1\right)\left(q^{2}+5 q+1\right)\right] q^{2 n-3}-\frac{1}{3} \varepsilon q^{\frac{2 n}{3}-1}\right\} \text {, }
\end{aligned}
$$

where $\varepsilon=1$ or 0 according as 3 does or does not divide $n$;

$$
M(1,1, n)=\left(q^{2}-1\right)\left\{\left(q^{2}+1\right)\left(q^{2}+q+1\right) q^{4 n-3}-\left[\left(q^{2}-1\right) n+(q+1)^{2}\right] q^{2 n-2}\right\} ;
$$

and

$$
\begin{aligned}
& M(1,2, n)=\left(q^{2}-1\right)\left\{\left(q^{4}+q^{2}+1\right)\left(q^{4}+q^{3}+q^{2}+q+1\right) q^{6 n-5}\right. \\
& -\left(q^{2}+1\right)\left(q^{2}+q+1\right)^{2} q^{4 n-4}-(q+1)^{2}\left(q^{2}+q+1\right) q^{3 n-3} \\
& \left.+\left[\frac{1}{2}\left(q^{2}-1\right) n^{2}+\frac{3}{2}\left(q^{2}-1\right)\left(2 q^{2}+2 q-1\right) n+\left(5 q^{3}+8 q^{2}+5 q-1\right)\right] q^{2 n-3}\right\} \\
& \text { Thus, we have, for large } n, \\
& M(1, n)=\left(1-q^{-1}\right) N(1, n)+(q-1) q^{n-1}, \\
& M(2, n)=\left(1-q^{-2}\right) N(2, n)-\frac{1}{2}\left(q^{2}-1\right)^{2} n q^{2 n-2}+O\left(q^{2 n}\right), \\
& M(3, n)=\left(1-q^{-3}\right) N(3, n)-\left(q^{2}+2 q+2\right)\left(q^{3}-1\right) q^{3 n-2}+O\left(n^{2} q^{2 n}\right), \\
& M(1,1, n)=\left(1-q^{-3}\right) N(1,1, n)-\left(q^{2}-1\right)^{2} n q^{2 n-2}+O\left(q^{2 n}\right),
\end{aligned}
$$

and

$M(1,2, n)=\left(1-q^{-5}\right) N(1,2, n)-\left(q^{4}-1\right)\left(q^{3}+2 q^{2}+q+1\right) q^{4 n-4}+O\left(q^{3 n}\right)$.

The above expressions illustrate Theorem 2 (and its exceptions) and also (5.11). 
The derivation of the above results is increasingly complicated. Each further computation, using this method, would require considerable calculation.

Finally, to illustrate Theorem 3 , we estimate $M(1, n, 2 n)$ for large $n$. For integers $i, r, s$ with $s \geqq 1$, let $N^{\prime}(i, r, s)$ denote the difference

$$
N(i, r, s)-q N(i, r, s-1) .
$$

Then from (4.1) and (5.6) we have

$$
\begin{aligned}
M(1, n, 2 n)=L(1, n, 2 n)= & N^{\prime}(1, n, 2 n)-\sum_{r=0}^{n-1} L(1, r, 2 n) N(0, n-r, 0) \\
& -\sum_{r=0}^{n-1} \sum_{s=0}^{2 n-1} L(1, r, s) N^{\prime}(0, n-r, 2 n-s) .
\end{aligned}
$$

Now, using an estimate for $N(1, n, 2 n-1)$, we have, for large $n$,

$$
N^{\prime}(1, n, 2 n)=N(1, n, 2 n)-q(q-1)^{-1}\left(q^{4 n(n+1)}-q^{2(n+1)(2 n-1)}+O\left(q^{4 n^{2}}\right)\right) .
$$

With the convention that $N^{\prime}(0, n-r, 0)$ is taken to mean $N(0, n-r, 0)$, it follows from (3.7) that, if $0 \leqq r \leqq n-1$ and $0 \leqq s \leqq 2 n$,

$$
L(1, r, s) N^{\prime}(0, n-r, 2 n-s) \leqq L(1, r, s) N(0, n-r, 2 n-s)=O\left(q^{T(1, r, s)}\right) .
$$

We show that if $0 \leqq r \leqq n-1$ and $0 \leqq s \leqq 2 n$ then

$$
T(1, r, s) \leqq 4 n^{2}+O(1)
$$

for large $n$, except when $r=n-1$ and $s=2 n$. If

$$
1 \leqq r \leqq n-1 \text { and } 1 \leqq s \leqq 2 n-1 \text {, }
$$

(6.4) follows from Lemma 3. Also, from the proof of Lemma 3, it is evident that, if $2 \leqq r \leqq n-2$ and $0 \leqq s \leqq 2 n$, then

$$
T(1, r, s) \leqq T(1, n-2,2 n)=4 n^{2}-2 n+1
$$

so that (6.4) holds in this case too. Again, if $0 \leqq r \leqq 1$ and $0 \leqq s \leqq 2 n$, we have

$$
\begin{aligned}
T(1, r, s) & =2(r+1)(s+1)+(n-r+1)(2 n-s+1) \\
& \leqq 2(r+1)+(n-r+1)(2 n+1), \quad \text { if } n \geqq 4 \\
& \leqq(n+1)(2 n+1)+2,
\end{aligned}
$$

since the function is decreasing with respect to $s$ for $n \geqq 4$. To complete the proof of (6.4), we observe that

$$
T(1, n-1,0)=2 n+2(2 n+1) \leqq 4 n^{2},
$$

for large $n$. Finally, from (4.10) and (3.1), we see that, for large $n$,

$$
\begin{aligned}
L(1, n-1,2 n) N(0,1,0) & =q\left(1-q^{-(2 n-1)}\right)(q-1)^{-1}\left(q^{2 n(2 n+1)}+O\left(q^{4 n^{2}}\right)\right) \\
& =q(q-1)^{-1} q^{2 n(2 n+1)}+O\left(q^{4 n^{2}}\right) .
\end{aligned}
$$


Hence, (6.3) yields, for large $n$,

$M(1, n, 2 n)=N(1, n, 2 n)-\left(1-q^{-1}\right)^{-1} q^{4 n(n+1)}-[(q+1)+o(1)] q^{2 n(2 n+1)-1}$, in accordance with Theorem 3 .

I wish to thank Dr. John Hunter for the invaluable assistance he has given me at all stages in the preparation of this paper.

\section{REFERENCES}

(1) L. Cartrtz, On factorable polynomials in several indeterminates, Duke Math. J., 2 (1936), 660-670.

(2) L. CarLITZ, The distribution of irreducible polynomials in several indeterminates, Illinois J. Math., 7 (1963), 371-375.

(3) L. CARLITZ, The distribution of irreducible polynomials in several indeterminates II, Canadian J. Math., 17 (1965), 261-266.

THE UNIVERSITY

GLASGOW, W.2

E.M.S.-B 